\title{
A MONOLITHIC 162-STAGE TWO-PART KNUDSEN PUMP FOR HIGH COMPRESSION RATIO
}

\author{
S. An*, N.K. Gupta, and Y.B. Gianchandani \\ Center for Wireless Integrated MicroSensing and Systems $\left(\mathrm{WIMS}^{2}\right)$, University of Michigan, \\ Ann Arbor, Michigan 48109, USA
}

\begin{abstract}
This paper reports on a 162-stage Knudsen pump, which is a motionless, thermomolecular pump, monolithically integrated into a single chip. The microchip is fabricated by a five mask silicon wafer process and has a footprint of $12 \times 15 \mathrm{~mm}^{2}$. It performs a self-evacuation of on-chip cavities, reducing pressures from 760 Torr to $\approx 7$ Torr. This is achieved with a power of $\approx 0.44 \mathrm{~W}$. The compression ratio of 109 is a $2 \times$ improvement over our previous best report, with a $3 \times$ reduction in power consumption. This performance is made possible by using a two-part design, which separately customizes stages for high and low pressure levels.
\end{abstract}

\section{INTRODUCTION}

First proposed and demonstrated by Knudsen in 1909 [1], the Knudsen pump, activated with thermal molecular pressure, has long been an appealing method for a vacuum pump. First, it has no moving parts [2]. Thermal gradient in a flow channel is the only driving force, so there is neither frictional loss nor mechanical failure. This greatly improves reliability and ensures long term operation. Second, it can potentially have small form factor. Using the miniaturization techniques of microelectromechanical systems (MEMS), compact Knudsen pumps have been reported [2-6].

Knudsen pumping is based on the phenomenon of thermal transpiration which occurs in a rarefied gas flow channel. If the hydraulic diameter of a flow channel is no larger than the mean free path (MFP) of gas molecules [2], i.e., the flow in the channel is confined to the free-molecular or transitional flow regimes, a thermal gradient results in flow from the cold end to the hot end. A monolithic Si implementation that used one such flow channel was reported in 2005, demonstrated a compression ratio of $\approx 2$ [2]. Because the compression ratio of a single-stage is limited by the temperature differential between the cold and hot ends, higher compression ratios can be achieved by multi-stage implementation. Mesoscale multistage Knudsen pumps have been reported [3, 5] in the past, but allow only a limited number of stages to be used.

In 2012 [6], our group reported a highly-packed multistage implementation, leveraging state-of-the-art silicon micromachining technology. It had 48 repetitions of a single type of stage for high compression ratio. The fabricated chip demonstrated the self-evacuation of on-chip cavities from 760 Torr to $<50$ Torr. However, this one-part approach is inadequate for a target vacuum level below about 50 Torr. The small height of the flow channel, where Knudsen pumping occurs, in the design $(0.15 \mu \mathrm{m})$ is suitable for initiating pumping from atmospheric pressure. However, when pumping is initiated from low pressure, the larger height of the channel can also provide similar compression with higher pumping speed, because the flow remains in free-molecular or transitional regime even for deeper flow channels that provide lower hydraulic resistance.

In this paper, we propose an improved, two-part design, which separately customizes stages for high and low pressure levels. Compression ratio and pumping speed are addressed in relation to the variation of narrow and wide channels at both pressure levels. Parametric design of a heater using finite element analysis (FEA) for low power heating is also described.

\section{DESIGN CONCEPT \\ Knudsen Pump Theory}

Thermal transpiration in a rarefied gas flow channel is described by thermal creep flow and viscous backflow [7]. The thermal gradient results in thermal creep flow along the channel walls from the cold end to the hot end; away from the walls, viscous backflow is caused by the pressure differential induced by the thermal creep flow. When the characteristic height of the flow channel is no larger than the MFP, the thermal creep flow is dominant over the viscous backflow; this establishes a pressure gradient in the same direction as the thermal gradient. In contrast, when the characteristic height of the flow channel is considerably larger than the MFP, the viscous backflow dominates, diminishing or eliminating the pressure gradient.

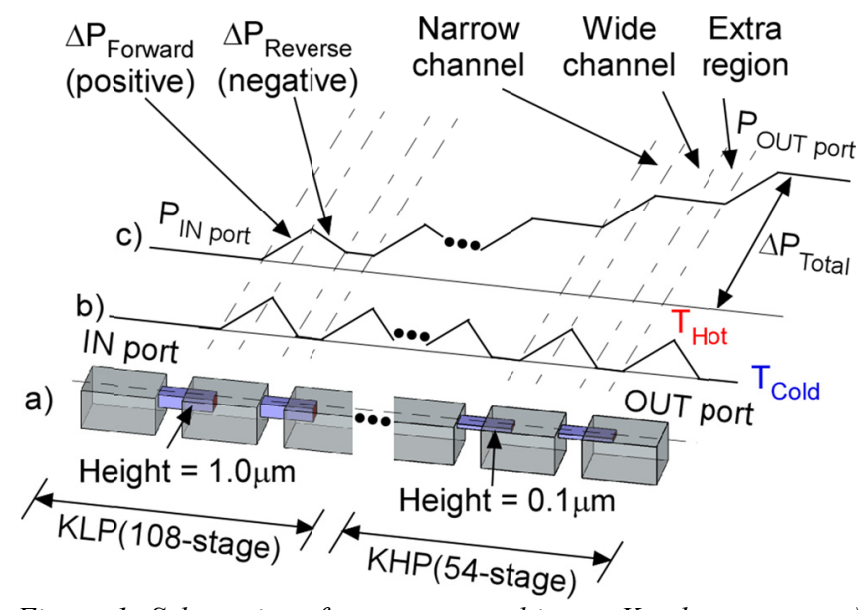

Figure 1: Schematics of a two-part multistage Knudsen pump. a) Serial cascading of KLP and KHP stages. b) Temperature profile along the dashed line in (a). c) Equilibrium pressure profile along the dashed line in (a). The dot-dash lines between (a)-(c) indicate simultaneous occurrences. KHP is from stage \#1 (OUT port) to \#54 and KLP is from stage \#55 to \#162 (IN port).

To achieve a large total pressure differential $\left(\Delta P_{\text {Total }}\right)$, multiple single-stage Knudsen pumps are serially cascaded (Fig. 1). Each stage includes a narrow channel and wide channel (Fig. 1a). The narrow channel, where the thermal creep flow is designed to be dominant, generates a forward pressure differential $\left(\Delta P_{\text {Forward }}\right)$ (Fig. 1c) under the applied temperature gradient along the intended forward path (Fig. 1b). In contrast, the wide channel, where the viscous backflow is designed to be dominant, is intended to restore the temperature from $\mathrm{T}_{\mathrm{Hot}}$ to $\mathrm{T}_{\text {Cold }}$ without a change in pressure. However, at low pressure, because the MFP increases to the extent that is comparable to the hydraulic diameter of the wide channel, a reverse pressure differential $\left(\Delta P_{\text {Reverse }}\right)$ is established in the wide channel, thereby reducing the net pressure differential $\left(\Delta P_{\text {Net }}=\Delta P_{\text {Forward }}-\Delta P_{\text {Reverse }}\right)$. This is mitigated by maximizing the dimension of the wide channel and by increasing the number of stages.

For the analytical design of micromachined Knudsen pumps, the Sharipov model [8] is an appropriate representative model for 
direct simulation Monte Carlo (DSMC) [9]. In a rectangular channel from the cold end to the hot end, the Sharipov model calculates the average mass flow rate of gas as:

$$
\frac{d M_{\text {avg }}}{d t}=\left(\frac{Q_{T} \Delta T}{T_{\text {avg }}}-\frac{Q_{P} \Delta P}{P_{\text {avg }}}\right) \frac{a^{2} b P_{\text {avg }}}{l} \sqrt{\frac{m}{2 k_{b} T_{\text {avg }}}}
$$

where $l$ is the length of the channel, $a$ is the height of the channel, $b$ is the width of the channel, $\Delta T$ is the temperature difference, $T_{a v g}$ is the average temperature, $\Delta P$ is the pressure difference, $P_{a v g}$ is the average of the pressure, $m$ is the mass of a single gas molecule, $k_{b}$ is the Boltzmann constant and $Q_{T}$ and $Q_{P}$ are, respectively, the thermal creep flow and Poiseuille flow (pressure-driven flow) coefficients that depend on the rarefaction parameter (inverse of Knudsen number). Over time, the gas flow approaches equilibrium, where there is no net mass flow, and the established pressure is given by:

$$
\Delta P_{\text {equilibrum }}=\frac{Q_{T}}{Q_{P}} \frac{\Delta T}{T_{\text {avg }}} P_{\text {avg }}
$$

$Q_{T}$ and $Q_{P}$ for each height/width ratio and rarefaction parameter can be found directly from the table or from an interpolation of the tabulated values. At a given pressure, the decrease of the channel height is accompanied by the increase of the $\Delta P_{\text {equilibrium }}$ and the decrease of the average mass flow rate, and vice versa.

Table I. The designed channel dimensions for KHP and KLP stages respectively, and the corresponding $M F P$ of $N_{2}$ gas at each pressure,

\begin{tabular}{|c|c|c|c|c|}
\hline $\begin{array}{c}\text { Two-part } \\
\text { architecture }\end{array}$ & $\begin{array}{c}\text { Narrow } \\
\text { channel } \\
\text { height } \\
(\mu \mathrm{m})\end{array}$ & $\begin{array}{c}\text { Wide } \\
\text { channel } \\
\text { height } \\
(\mu \mathrm{m})\end{array}$ & $\begin{array}{l}\text { Pressure } \\
\text { (Torr) }\end{array}$ & $\begin{array}{l}\text { MFP } \\
(\mu \mathrm{m})\end{array}$ \\
\hline \multirow{3}{*}{$\begin{array}{c}\text { KHP } \\
\text { (760Torr } \\
-50 \text { Torr) }\end{array}$} & \multirow{3}{*}{0.1} & \multirow{3}{*}{$>30$} & 760 & 0.07 \\
\hline & & & 200 & 0.26 \\
\hline & & & 50 & 1.02 \\
\hline $\begin{array}{c}\text { KLP } \\
\text { ( } \leq 50 \text { Torr })\end{array}$ & 1.0 & $>50$ & $\frac{50}{1}$ & $\begin{array}{c}1.02 \\
51.10\end{array}$ \\
\hline
\end{tabular}
calculated at $300 \mathrm{~K}$.

\section{Two-part Architecture}

Due to the increase in the MFP resulting from the decrease in pressure level, the narrow channel can be enlarged at low pressure with an insignificant reduction of $\Delta P_{\text {Forward }}$, as long as its height is no larger than the MFP. Pumping speed can be considerably improved with an enlarged channel height, because mass flow rate is quadratically proportional to the channel height as shown in Eq. (1). In addition, the wide channel height needs to be significantly larger than the MFP at both high and low pressures to minimize $\Delta P_{\text {Reverse }}$. Hence, to enhance pumping speed and compression ratio at both high and low pressures, a two-part architecture (Fig. 1) is used: the Knudsen high-pressure part (KHP), located downstream, is designed for operating near atmospheric pressure, and the Knudsen low-pressure part (KLP), located upstream, is designed for $\leq 50$ Torr, as summarized in Table I. In the KHP, which pumps down from 760 Torr to 50 Torr, the narrow channel height is $0.1 \mu \mathrm{m}$, which is comparable to an MFP of $0.07 \mu \mathrm{m}$ at 760 Torr. In the KLP, which pumps down from 50 Torr to sub-Torr, the narrow channel height is $1.0 \mu \mathrm{m}$, which is comparable to the MFP of $1.0 \mu \mathrm{m}$ at 50 Torr. The wide channel height of the KHP is larger than $30 \mu \mathrm{m}$ for minimum $\Delta P_{\text {Reverse }}$ from 760 Torr to 50 Torr. The wide channel height of the KLP is maximized to be as deep as fabrication permits. In this generation of Knudsen pumps, the wide channel height of both KHP and KLP is equal to $\approx 50 \mu \mathrm{m}$ for the simplification of fabrication and mask design.

\section{Parametric Design of Heater Structure}

The length of the narrow channel in the device structure (Fig. $2 \mathrm{f}$ ) is one of the dominant factors determining the thermal isolation from the heater to the thermally ground. By varying structural parameters such as width, length, and thickness, the dominant dimensional parameters for low power heating are extracted and fine-tuned using FEA. Parametric FEA enables lower power operation in this generation of Knudsen pumps.

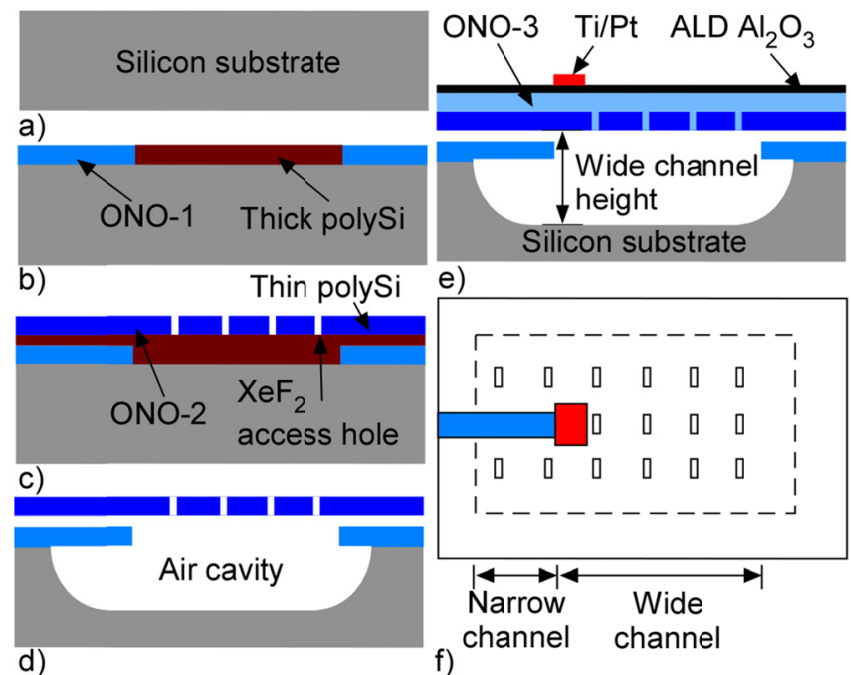

Figure 2: The five mask fabrication process and sectional views of structure. a-e) Side view of each fabrication process step: a) silicon wafer, b) (Mask I) deposition and patterning of ONO-1 and (Mask II) deposition and patterning of thick polySi for KLP narrow channel, c) (Mask III) deposition \& patterning of thin polySi for KHP narrow channel and (Mask IV) deposition and patterning of $\mathrm{ONO}-2$, d) $\mathrm{XeF}_{2}$ etch of polySi and air cavity, and e) deposition of ONO-3 and atomic layer deposited (ALD) $\mathrm{Al}_{2} \mathrm{O}_{3}$ for sealing and (Mask V) lift-off of Ti/Pt heater and leads. f) Top view of complete structure.

\section{FABRICATION}

\section{Microfabrication Process}

To implement the multistage Knudsen pump, a simple and high yield single wafer process is used; it uses five masking steps (Fig. 2). Important features in the channel fabrication are: 1) thin (sacrificial) polysilicon is used to define narrow channels in the KHP stages (Fig. 2c); 2) both thin and thick (sacrificial) polysilicon layers are used to define narrow channels for the KLP stages; 3) a cavity is etched in bulk silicon to increase the height of the wide channel (Fig. 2e); and 4) the cavity, in the left side under the heater, also defines the length of the narrow channel (Fig. 2f); and 5) stress compensated oxide-nitride-oxide (ONO) layers, ONO-1 and ONO-2, are used to define the walls of the narrow channel. A Ti/Pt heater, located at the end of the narrow channel, generates the intended temperature gradient for the $\Delta P_{\text {Forward }}$ in the narrow channel. The temperature gradient in the wide channel occurs, because the wide channel is linking the hot end of one narrow channel and the cold end of the next narrow channel. Increasing the height of the wide channel minimizes $\Delta P_{\text {Reverse }}$ at low pressures. At each step in the process, the thickness and residual stress of each layer are monitored (Table 2). ONO layers are designed to have a mild tensile stress to avoid a buckling of the suspended diaphragm due to compressive residual stress. 
Table 2: Summary of the approximate values of the thickness and residual stress of each layer.

\begin{tabular}{|c|c|c|}
\hline Layer & $\begin{array}{c}\text { Thickness } \\
(\boldsymbol{\mu m})\end{array}$ & $\begin{array}{c}\text { Residual stress } \\
(\mathrm{MPa})\end{array}$ \\
\hline $\mathrm{ONO}-1$ & $0.5 / 0.2 / 0.5$ & +42.5 \\
\hline $\mathrm{ONO}-2$ & $0.5 / 0.2 / 0.5$ & +42.5 \\
\hline $\mathrm{ONO}-3$ & $0.7 / 0.5 / 0.7$ & +42.1 \\
\hline $\mathrm{Thin} /$ thick poly & $0.1 / 0.7$ & - \\
\hline $\mathrm{ALD} \mathrm{Al} \mathrm{O}_{3}$ & 0.2 & +304 \\
\hline $\mathrm{Ti} / \mathrm{Pt}$ & $0.025 / 0.1$ & - \\
\hline
\end{tabular}

Additional challenges in microfabrication compared to our Knudsen pump in [6] are as follows: increased wide channel height using a higher density of $\mathrm{XeF}_{2}$ access holes for reduced $\Delta P_{\text {Reverse }}$; a reduced thickness of sealing layers for higher thermal isolation; and a decreased Ti/Pt-heater size by reducing winding-pattern linewidth, also for higher thermal isolation.

a)
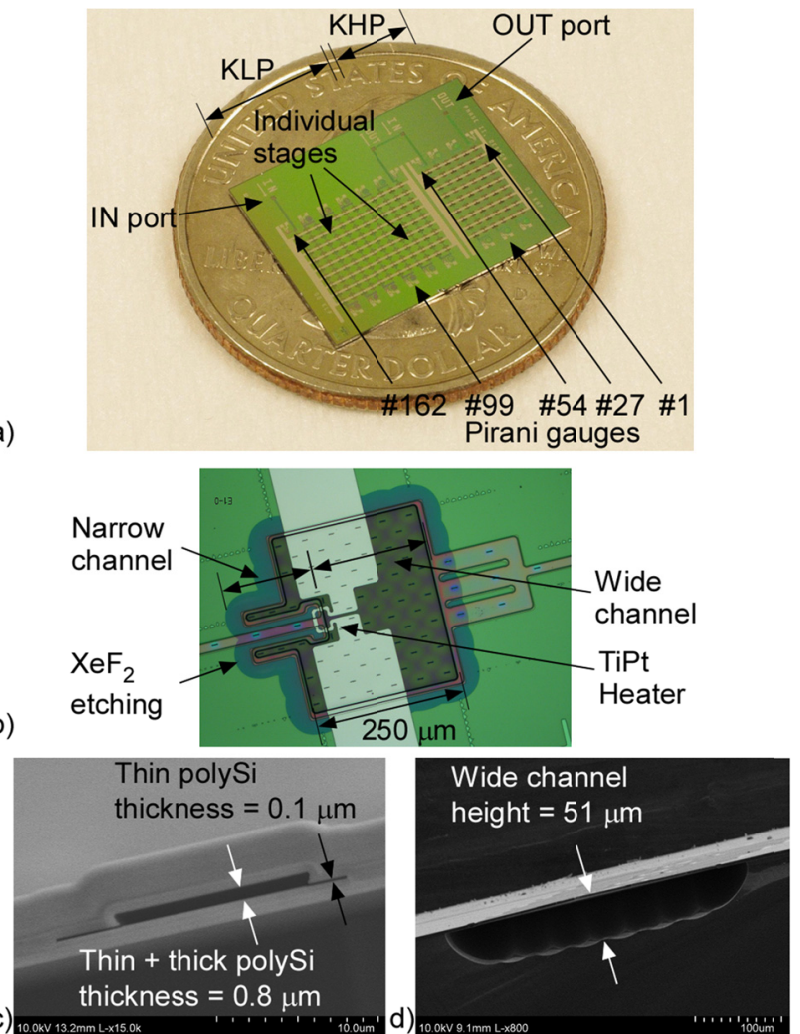

Figure 3: Photographs of the Knudsen pump as fabricated. a) The full die. b) Top view of one stage. c) SEM of the cross section of a narrow channel in the KLP. d) SEM of the cross section of a wide channel in both KLP and KHP.

\section{Fabrication Results}

The fabricated chip (Fig. 3a) has a footprint of $12 \times 15 \mathrm{~mm}^{2}$. A typical yield is $60-80 \%$. The KHP and KLP are hydraulically connected, using sacrificially etched polysilicon layers. Pirani gauges for the in situ measurement of vacuum levels are located on both sides of the pumping stages.

The length of the narrow channel with a temperature gradient is defined by $\mathrm{XeF}_{2}$ gas etching of the bulk silicon (Fig. 3b). The resistive heaters, required for the Knudsen pumping stages, are shaped with winding patterns of $5 \mu \mathrm{m}$ width.

Narrow channels (Fig.3c) are formed by etching sacrificial polySi layers. For the narrow channel of the KHP, only a thin polySi layer of $0.1 \mu \mathrm{m}$ thickness is used. However, for the narrow channel of the KLP, both thin and thick polySi layers of $0.8 \mu \mathrm{m}$ are used as sacrificial layers.

The wide channel height (Fig. 3d) is defined by the etching depth of the bulk silicon, using $\mathrm{XeF}_{2}$ gas. The density of the $\mathrm{XeF}_{2}$ access holes (Fig. 2c) and etching time determine the height of the wide channel. The $\mathrm{XeF}_{2}$ access hole is designed to be $1 \mu \mathrm{m} \times 10$ $\mu \mathrm{m}$ in size and is hermetically sealed by PECVD ONO-3 and ALD $\mathrm{Al}_{2} \mathrm{O}_{3}$ layers.

\section{TEST RESULTS AND DISCUSSION Test Preparation}

The fabricated chip is wire-bonded for electrical connections. The base of the Knudsen pump is placed in direct contact with a test chamber for the thermal grounding of the silicon substrate to the room temperature laboratory environment. The operating medium gas is air. Prior to the activation of the Knudsen pump, the five Pirani gauges $(\approx 125 \Omega)$, including the IN port and OUT port, are calibrated from high to low pressure levels (Fig. 4a). The IN port of the KLP is sealed, while the OUT port of the KHP is opened and vented to ambient pressure. The ambient pressure in the test chamber is controlled externally. By applying a constant current source of $4 \mathrm{~mA}$, the fractional changes in the resistance of each Pirani gauge are recorded at various pressure levels, representing the vacuum levels in nearby pumping stages upon pumping operation [6].

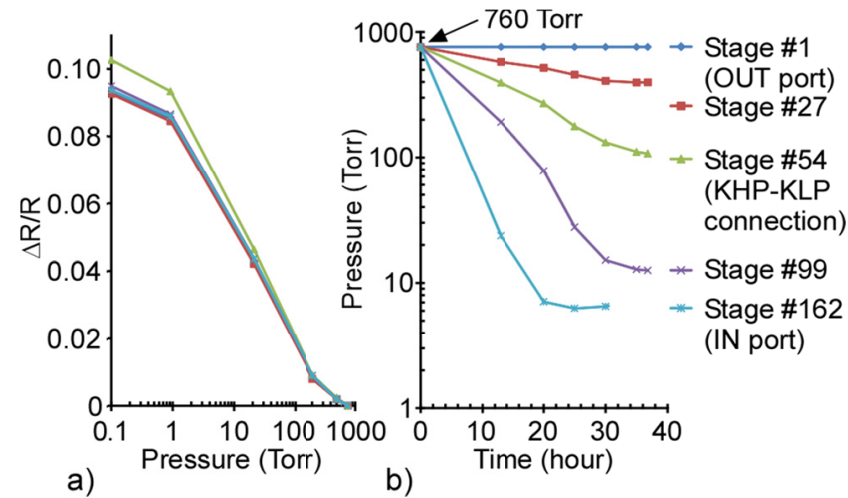

Figure 4: Pirani gauge calibration and time response of pressure a) Variation in the fractional change of the resistance of the five Pirani gauges. b) Measured time response of pressure levels in each stage, pumping down from 760 Torr, using an applied total power of $\approx 0.44 \mathrm{~W}$ to both KHP and KLP.

\section{Pumping Performance}

The pressure at the IN port approaches saturation in about 20 hours (Fig. 4b). In the case of the KHP-KLP connection stage, pressure saturation takes about 40 hours.

When only the KLP operates, pressure is reduced from 50 Torr to $\approx 2$ Torr, with an applied power of $\approx 0.3 \mathrm{~W}$ (Fig. $5 \mathrm{a}$ ). When both KLP and the KHP operate, pressure is reduced from 760 Torr to $\approx 7$ Torr, with an applied power of $\approx 0.44 \mathrm{~W}$ (Fig. 5b). This pump achieves a compression ratio of 109 when both KLP and the KHP are operating at 760 Torr.

\section{Discussion}

The KLP is composed of 108 stages while the KHP is composed of 54 stages. Due to the large height of the narrow channel in the KLP compared to the MFP at 760 Torr, the pressure is only reduced from 760 Torr to 150 Torr with KLP-only operation (Fig. 5a). In contrast, as shown in the simultaneous operation of KHP and KLP, the KHP can pump down from 760 Torr to about 
100 Torr (Fig. 5b), using a smaller number of stages than the KLP. In this two-part approach, the KHP, using the smaller height of the narrow channel suitable for initiation of pumping from atmospheric pressure, allows a greater compression ratio per stage and consequently operates with a smaller number of stages than the KLP at high pressure.

The KHP can also pump down from 50 Torr to less than 10 Torr (Fig. 5b). However, due to the dependence of mass flow rate upon the narrow channel height, the pumping time is longer. Using the larger narrow channel of KLP, mass flow rate is increased by about $64 \times$, which is simply the square of the ratio of the fabricated narrow channel height of KLP and KHP, as shown in Eq. (1). This is one of the reasons why the pressure at the IN port saturates twice as fast as the pressure at the KHP-KLP connection stage (Fig. 4b).

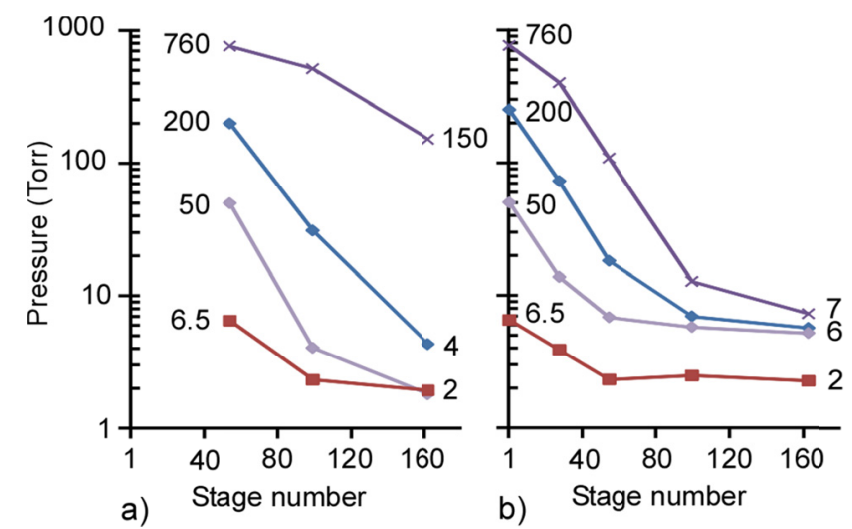

Figure 5: Measured pressure distribution in the selected five stages. a) Only KLP operating for pumping. b) Both KLP and KHP operating for pumping. The IN port is sealed while the OUT port is open to a test chamber whose pressure is controlled.

\section{Benchmarking}

Benchmarking shows that the resulting compression ratio of 109 exceeds the highest [6] by a factor of $2 \times$, with $3 \times$ power efficient (Fig. 6). The integration of 162 stages into one silicon chip, using the simple silicon-based micromachining, and two-part approach, separately customizing KHP/KLP stages, enabled this substantially higher performance.

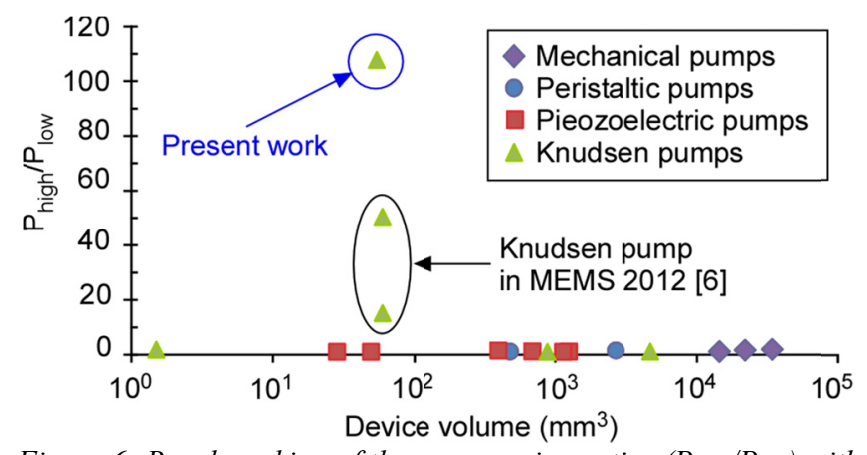

Figure 6: Benchmarking of the compression ratios $\left(P_{\text {high }} / P_{\text {low }}\right)$ with other pumps reported in literature to-date [2, 6, 10-11]. The present work achieves 760 Torr/7 Torr using both KHP and KLP.

\section{CONCLUSION}

A 162-stage Knudsen pump for high compression ratio, with an improved two-part (KHP and KLP) approach, is studied. Using a monolithic integration with a single-wafer microfabrication process, the designed highly-packed stages are serially cascaded in a small footprint of $12 \times 15 \mathrm{~mm}^{2}$. The pumping performance is measured in situ at five stages including the IN port and OUT port, and at various ambient pressures, using the calibration of Pirani gauges. Using KHP and KLP together, the fabricated Knudsen pump demonstrated the self-evacuation of on-chip cavities from 760 Torr to $\approx 7$ Torr, with an input power of $\approx 0.44 \mathrm{~W}$. Compared to our last reported micropump [6], this shows a $2 \times$ improvement in compression ratio, using $3 \times$ smaller power consumption. The substantial contributions are from the two-part Knudsen pumping architecture together with the monolithic integration of the increased number of stages by silicon-based micromachining technology. In the future, the further refinement of the heater and flow-channel structure, along with the microfabrication process, could enhance the pumping performance to higher vacuum levels with even lower power consumption in a smaller footprint.

\section{ACKNOWLEDGEMENT}

The study is supported in part by the Microsystems Technology Office of the Defense Advanced Research Projects Agency High-Vacuum Program (DARPA Hi-Vac). Facilities used for this research include the Lurie Nanofabrication Facility (LNF) operated by the Solid-State Electronics Laboratory (SSEL) and the University of Michigan.

\section{REFERENCES}

[1] M. Knudsen, "Eine Revision der Gleichgewichtsbedingung der Gase. Thermische Molekularstromung," Annalen der Physik, Leipzig, vol. 336, no. 1, pp. 205-229, 1909. (in German).

[2] S. McNamara, and Y.B. Gianchandani, "On-Chip Vacuum Generated by a Micromachined Knudsen Pump," $J$. Microelectromech. Syst., vol. 14, no. 4, pp. 741-746, 2005.

[3] M. Young, Y.L. Han, E.P. Muntz, and G. Shiflett, "Characterization of a Radiantly Driven Multistage Knudsen Compressor," Proc. ASME Int. Mech. Engin. Congress (IMECE'03), pp. 393-400, 2003.

[4] K. Pharas, and S. McNamara, "Knudsen Pump Driven by a Thermoelectric Material," J. Micromech. Microeng, vol. 20, no. 12, pp. 125032, 2010.

[5] N.K. Gupta, and Y.B. Gianchandani, "Porous Ceramics for Multistage Knudsen Micropumps - Modeling Approach and Experimental Evaluation," J. Micromech. Microeng, vol. 21, no. 9, pp. 095029, 2011.

[6] N.K. Gupta, S. An, and Y.B. Gianchandani, "A Monolithic 48-Stage Si-micromachined Knudsen Pump for High Compression Ratios," Proc. IEEE Micro Electro Mechanical Systems (MEMS'12), pp. 152-155, 2012.

[7] L. Loeb, The Kinetic Theory of Gases, McGraw Hill, pp. 355-359, 1934.

[8] F. Sharipov, "Non-isothermal Gas Flow through Rectangular Microchannels," J. Micromech. Microeng., vol. 9, no. 4, pp. 394-401, 1999.

[9] N.K. Gupta, N. D. Masters, W. Ye, Y.B. Gianchandani, "Gas Flow in Nano-Channels: Thermal Transpiration Models with Application to a Si-Micromachined Knudsen Pump," Proc. IEEE Transducers '07, Lyon, France, pp. 2329-2332, 2007.

[10] H. Kim, A.A. Astle, K. Najafi, L.P. Bernal, and P.D. Washabaugh, "A Fully Integrated High-Efficiency Peristaltic 18-Stage Gas Micropump with Active Microvalves," Proc. IEEE Micro Electro Mechanical Systems (MEMS'07), pp. 127-130, 2007.

[11] D.J. Laser, and J.G. Santiago, “A Review of Micropumps,” J. Micromech. Microeng., vol. 14, no. 6, pp. R35-R64, 2004.

\section{CONTACT}

*S. An, tel: +1-734-717-7853; sdan@umich.com 